Martin Rohacek

Janet Buatsi

Zsolt Szucs-Farkas

Birgit Kleim

Heinz Zimmermann

Aristomenis Exadaktylos

Christoforos Stoupis

\title{
Ordering CT pulmonary angiography to exclude pulmonary embolism: defense versus evidence in the emergency room
}

Received: 29 November 2011

Accepted: 22 April 2012

Published online: 15 May 2012

(C) Copyright jointly held by Springer and ESICM 2012

Electronic supplementary material

The online version of this article (doi:10.1007/s00134-012-2595-z) contains supplementary material, which is available to authorized users.

M. Rohacek ( $)$ J. Buatsi ·

H. Zimmermann - A. Exadaktylos

Department of Emergency Medicine, Inselspital, University Hospital Bern, Freiburgstrasse, 3010 Bern, Switzerland e-mail: martin.rohacek@gmail.com

Tel.: +41-31-6322111

Fax: +41-31-6324757

Z. Szucs-Farkas · C. Stoupis

Department of Radiology, Inselspital,

University Hospital Bern, Bern, Switzerland

B. Kleim

Department for Clinical Psychology, University Zürich, Zurich, Switzerland
Abstract Purpose: To identify reasons for ordering computed tomography pulmonary angiography (CTPA), to identify the frequency of reasons for CTPA reflecting defensive behavior and evidence-based behavior, and to identify the impact of defensive medicine and of training about diagnosing pulmonary embolism (PE) on positive results of CTPA. Methods: Physicians in the emergency department of a tertiary care hospital completed a questionnaire before CTPA after being trained about diagnosing PE and completing questionnaires. Results: Nine hundred patients received a CTPA during 3 years. For 328 CTPAs performed during the 1-year study period, 140 (43\%) questionnaires were completed. The most frequent reasons for ordering a CTPA were to confirm/rule out PE (93\%), elevated D-dimers (66\%), fear of missing PE (55\%), and Wells/simplified revised Geneva score $(53 \%)$. A positive answer for "fear of missing PE" was inversely associated with positive CTPA (OR
0.36, $95 \%$ CI 0.14-0.92, $p=0.033$ ), and "Wells/simplified revised Geneva score" was associated with positive CTPA (OR 3.28, $95 \%$ CI $1.24-8.68, p=0.017)$. The proportion of positive CTPA was higher if a questionnaire was completed, compared to the 2-year comparison period (26.4 vs. $14.5 \%$, OR 2.12, $95 \%$ CI $1.36-3.29, p<0.001)$. The proportion of positive CTPA was nonsignificantly higher during the study period than during the comparison period (19.2 vs. $14.5 \%$, OR 1.40, $95 \%$ CI $0.98-2.0, p=0.067)$. Conclusion: Reasons for CTPA reflecting defensive behavior-such as "fear of missing PE" - were frequent, and were associated with a decreased odds of positive CTPA. Defensive behavior might be modifiable by training in using guidelines.

Keywords Computed tomography . Pulmonary embolism - Defensive medicine $\cdot$ Fear

\section{Introduction}

Acute pulmonary embolism (PE) is a disease with an annual incidence of approximately 3-6 cases per 10,000 persons in the general population $[1,2]$. Symptoms of PE range from no symptoms at all to chest pain or dyspnea and can result in pulseless electrical activity or cardiac arrest. Depending on the clinical presentation and severity of PE, the case fatality rate ranges from less than $1 \%$ to about $60 \%$ [3]. Diagnostic tools such as the D-dimer assay and computed tomography pulmonary angiography (CTPA) are recommended in the diagnostic workup of suspected PE [4]. The value of these tests, however, depends on the clinical pretest probability, which can be estimated by calculating validated Wells and simplified revised Geneva scores 
(Wells/Geneva score, see table in the supplementary appendix) [5, 6].

Surveys suggest that defensive medicine plays a role in ordering computed tomography examinations (CT). Defensive medicine is defined as the ordering of treatments, tests, and procedures with the primary aim of protecting the physician from liability rather than of substantially furthering the patient's diagnosis or treatment [7]. In a 2005 survey, Studdert et al. [8] showed that $93 \%$ of questioned physicians in Pennsylvania reported that they practiced defensive medicine, and that $63 \%$ of emergency department physicians ordered radiological tests that were not indicated. A 2008 survey from Massachusetts revealed that $23 \%$ of all types of CTs were ordered for defensive reasons [9]. However, European data about defensive medicine and data about reasons for practicing defensive medicine are scarce. In this study, we therefore aimed to evaluate physicians' motivations for ordering a CTPA. We hypothesized that defensive factors, such as fear of missing PE or demand from the patient or his/her relatives, could be a common reason for ordering an unnecessary CTPA, and that documentation of motivations for ordering a CTPA, coupled with appropriate training in medical decision making to diagnose PE, might influence the behavior of physicians and direct them towards evidence-based medicine.

\section{Methods}

Patients and physicians

This study analyzed the reasons for ordering CTPA, the frequency of reasons for CTPA reflecting defensive behavior and evidence-based behavior, and the impact of defensive medicine and of training about diagnosing pulmonary embolism (PE) on positive results of CTPA (positive CTPA, i.e., evidence of central, paracentral, segmental, or subsegmental embolism of the pulmonary artery in CTPA). For this purpose, from 1 February 2010 to 31 January 2011, all physicians of our tertiary care hospital's emergency department, caring for about 30,000 patients per year, were asked to complete an anonymous questionnaire and to assign the Wells/Geneva score on a voluntary basis before ordering CTPA, after being trained in diagnosing PE. We compared the proportion of positive CTPAs during the study period with a comparison period. Therefore, frequencies and results of CTPAs performed in the 2 years before the study period were also recorded. Thus, all patients who received CTPA for suspected PE at the emergency department from 1 January 2008 through 31 January 2011 were included, regardless of other additional reasons for the same CT (i.e., trauma, suspected cancer, aortic dissection, or pulmonary diseases). All patients were identified in our internal database.
Characteristics were recorded from all included patients. All patients were examined with a 16-row CT system (Somatom Sensation 16, Siemens, Erlangen, Germany). All CTPAs were reviewed by at least two radiologists, one of them a board certified radiologist with a minimum of 5 years of experience in CTPA. To measure D-dimers, the enzyme-linked fluorescent immunoassay VIDAS $\odot$ (bioMérieux, Marcy l'Etoile, France) was used, with a cutoff value of $500 \mathrm{ng} / \mathrm{mL}$.

At our institution, every decision to perform a CTPA has to be discussed with a senior physician, with a minimum clinical experience of 5 years. Physicians working at the emergency department change regularly. Therefore, we evaluated the number of senior and assistant physicians working before and during the study from monthly duty rosters.

\section{Questionnaire}

The questionnaire (see figure in the supplementary appendix) comprised the following information: First, the physician (senior or assistant) ordering a CTPA filled in the pretest probability of PE (low, intermediate, high), regardless of whether the probability was estimated by using a score or by the physician's clinical experience. Second, the physician marked suggested reasons for ordering the CTPA on a scale from 0 (not relevant at all) to 10 (highly relevant). Suggested reasons to select were the Wells/Geneva score, elevated D-dimers, to confirm/ rule out $\mathrm{PE}$, to expedite diagnosis, request of a consultant, request of the family doctor, request of a senior physician, request of the patient or his/her relatives, fear of missing $\mathrm{PE}$, fear of being sued, and difficulty in evaluating medical history. The reason "Wells/Geneva score" reflected evidence-based behavior, and "fear of missing PE", "request of the patient/relatives", and "fear of being sued", reflected defensive behavior. "Fear of missing PE" was defined as evidence that CTPA was ordered because of anxiety of missing PE, although the physician did not really believe that the patient suffered from PE and that a CTPA was truly needed.

To clarify potential areas of confusion, the questionnaire was presented to a group of physicians prior to distribution. A sheet with the Wells/Geneva score calculation directions was provided with the questionnaire.

\section{Training}

At the beginning of the study period, or during the first week working at the emergency department, every physician received a one-to-one training session about estimating pretest probabilities using the Wells/Geneva score and about diagnosing PE using the D-dimer test and CTPA (i.e., that a non-elevated D-dimer test safely rules 
out PE in case of low or intermediate pretest probability). Instructions how to complete the questionnaire were given, and the meaning of "fear of missing PE" was explained. Physicians were reminded each week to follow these guidelines and to complete the questionnaire.

\section{Statistical analysis}

For all statistical analysis, the statistical package $\mathrm{R}$ (The $\mathrm{R}$ Foundation for Statistical Computing Version 2.12.2) was used. The outcome measure was positive CTPA. Fisher's exact test was used to calculate $p$ values to compare the characteristics of the patients who received a CTPA. A difference with a $p$ value of less than 0.05 was considered to be significant. In order to investigate the relation of variables to positive CTPA, two multivariable logistic regression models were applied: In the first model, independent variables were "calculated Wells/ Geneva score" and positive answers for the reasons "Wells/Geneva score", "elevated D-dimers", "fear of missing PE", "request of the patient/relatives", and "fear of being sued". A positive answer was defined as any marked number from 1 to 10 on the scale from 0 to 10 . If a scale was left empty, 0 was assumed. In the second model, independent variables were linear scale parameters of the reasons "Wells/Geneva score", "elevated D-dimers", "fear of missing PE", "request of the patient/ relatives", and "fear of being sued". For the linear scale parameters, the odds ratio (OR) was expressed as the ratio of the odds from the $3 \mathrm{rd}$ to the 1 st quartile ( 75 and 25 percentile, respectively), representing a typical above average to a typical below average value. Logistic regression was used to investigate the relation of other variables to a positive CTPA. Independent variables were time periods (comparison period, study period), completed questionnaire, and pretest probability for PE (high vs. low). In an additional analysis of CTPAs evaluating patients for PE only, independent variables were time periods and completed questionnaire.

\section{Ethics statement}

The study was approved by the institutional review board and was conducted in compliance with the Declaration of Helsinki. The institutional review board approved the waiver of informed consent.

\section{Results}

Patients and physicians

Data from all included patients were available. From 1 January 2008 through 31 January 2011, a total of 1,119 CT exams of the chest were performed at the emergency department. Of these, 900 were CTPAs. Of 328/900 CTPAs performed during the study period, $140(43 \%)$ questionnaires were completed (Fig. 1). Characteristics of patients who received a CTPA are shown in Table 1. During the study period, there were significantly more patients with a sign of deep vein thrombosis than during the comparison period. In the group of patients with a completed questionnaire, there were significantly more patients with a sign of deep vein thrombosis and more patients on oral contraceptives than during the comparison period and than in the group of patients without a completed questionnaire during the study period. There was a median of 19 (range 16-22) senior physician positions, and there were 9 and 27 changes during the study and comparison period, respectively. Seven senior physicians worked through all 3 years. There was a median of 21 (range 18-29) assistant physician positions available. Assistant physicians rotated every 6-12 months.
Fig. 1 Computed tomograms during study and comparison period. $C T$ computed tomography, CTPA computed tomography pulmonary angiography, $P E$ pulmonary embolism

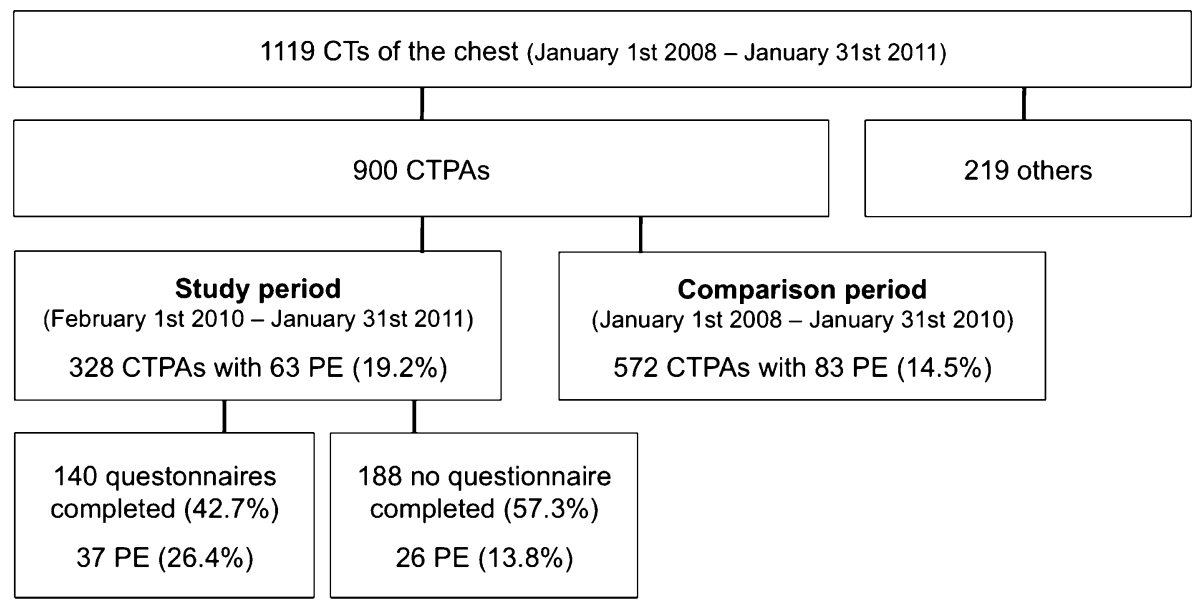


Table 1 Characteristics of patients

\begin{tabular}{|c|c|c|c|c|c|c|c|}
\hline Characteristics & $\begin{array}{l}\text { Comparison } \\
\text { period } \\
n=572\end{array}$ & $\begin{array}{l}\begin{array}{l}\text { Study } \\
\text { period_all }\end{array} \\
n=328\end{array}$ & $p$ value* & $\begin{array}{l}\text { Study period- } \\
\text { completed } \\
\text { questionnaire } \\
n=140\end{array}$ & $p$ value $^{\dagger}$ & $\begin{array}{l}\text { Study period- } \\
\text { no completed } \\
\text { questionnaire } \\
n=188\end{array}$ & $p$ value \\
\hline Age-mean years $(\mathrm{SD})$ & $61.3(16.5)$ & $59.6(17.3)$ & & $57.9(19.0)$ & & $60.9(15.9)$ & \\
\hline Male sex $-n(\%)$ & $319(56)$ & $179(55)$ & 0.84 & $77(55)$ & 0.92 & $102(54)$ & 0.91 \\
\hline Active cancer $-n(\%)$ & $94(16)$ & $42(13)$ & 0.15 & $17(12)$ & 0.24 & $25(13)$ & 0.87 \\
\hline History of thromboembolism- $n(\%)$ & $60(11)$ & $38(12)$ & 0.66 & $13(9)$ & 0.76 & $25(13)$ & 0.30 \\
\hline Hospitalization $^{\mathrm{a}}-n(\%)$ & $47(8)$ & $30(9)$ & 0.62 & $13(9)$ & 0.73 & $17(9)$ & 1 \\
\hline Surgery ${ }^{\mathrm{b}}-n(\%)$ & $26(5)$ & $22(7)$ & 0.17 & $9(6)$ & 0.38 & $13(7)$ & 1 \\
\hline Oral contraceptives- $n(\%)$ & $13(2)$ & $10(3)$ & 0.51 & $9(6)$ & 0.024 & $1(1)$ & 0.003 \\
\hline Dyspnea-n $(\%)$ & $307(54)$ & $172(52)$ & 0.73 & $80(57)$ & 0.51 & $92(49)$ & 0.15 \\
\hline Chest pain-n $(\%)$ & $309(54)$ & $192(59)$ & 0.21 & $92(66)$ & 0.013 & $100(53)$ & 0.024 \\
\hline Tachycardia ${ }^{\mathrm{c}}-n(\%)$ & $140(25)$ & $79(24)$ & 0.94 & $34(24)$ & 1 & $45(24)$ & 1 \\
\hline Sign of deep vein thrombosis ${ }^{\mathrm{d}}-n(\%)$ & $40(7)$ & $37(11)$ & 0.035 & $25(18)$ & $<0.001$ & $12(6)$ & 0.001 \\
\hline Cardiac arrest/CPR- $n(\%)$ & $20(4)$ & $11(3)$ & 1 & $4(3)$ & 1 & $7(4)$ & 0.76 \\
\hline Hemoptysis- $n(\%)$ & $19(3)$ & $8(2)$ & 0.55 & $6(4)$ & 0.61 & $2(1)$ & 0.08 \\
\hline \multicolumn{8}{|l|}{ D-dimers- $n(\%)$} \\
\hline Elevated $^{\mathrm{e}}$ & $350(61)$ & $217(66)$ & 0.27 & $107(77)$ & 0.002 & $110(59)$ & 0.002 \\
\hline No D-dimer & $187(33)$ & $90(28)$ & & $26(19)$ & & $73(37)$ & \\
\hline \multicolumn{3}{|c|}{$\begin{array}{l}C P R \text { cardiopulmonary resuscitation } \\
* p \text { value for group of patients during comparison period compared } \\
\text { with the group of all patients during study period; } p \text { value for the } \\
\text { group with completed questionnaire, compared with the group } \\
\text { during comparison period; }{ }^{\star} p \text { value for group without questionnaire } \\
\text { during study period compared with group with completed ques- } \\
\text { tionnaire. Fisher's exact test was used to calculate } p \text { values; a }\end{array}$} & \multicolumn{5}{|c|}{$\begin{array}{l}\text { difference with a } p \text { value of less than } 0 \text {. } \\
\text { significant } \\
\text { a Hospitalization during previous month } \\
\text { b Surgery during previous month } \\
\text { c }>100 \text { beats/min } \\
\text { d Pain or swelling of a limb } \\
\text { e }>500 \text { n } / \mathrm{mL}\end{array}$} \\
\hline
\end{tabular}

Fig. 2 Positive answer is defined as any marked number from 1 to 10 on a scale from 0 to 10 in the questionnaire

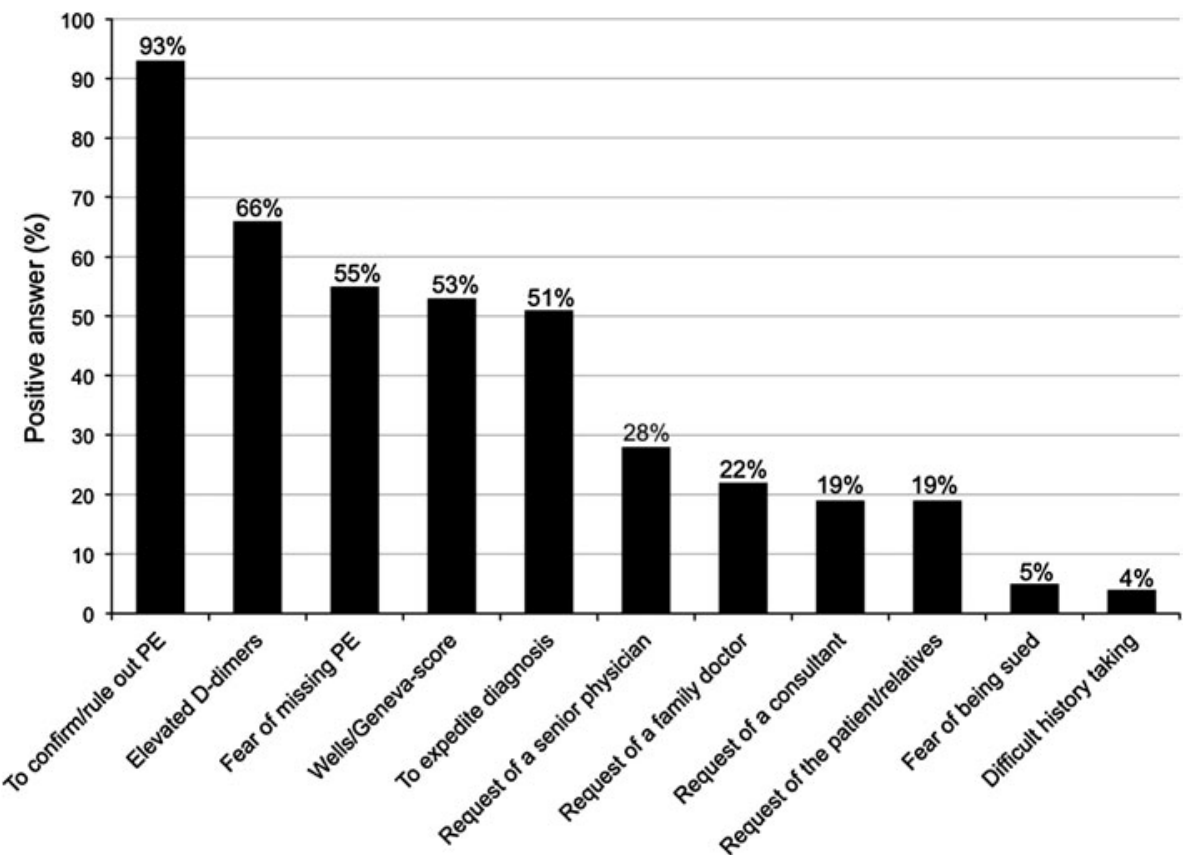

Reasons for ordering a CTPA

The three most common reasons with a positive answer in the questionnaires were "confirm/rule out PE" (in $93 \%$ of all questionnaires), "elevated D-dimers" (66\%), and "fear of missing PE" (55 \%). "Wells/Geneva score" was declared in $53 \%$ of questionnaires (Fig. 2). In multivariable logistic regression models, a positive answer and scale parameters for "fear of missing PE" were inversely associated with positive CTPA, whereas a positive answer 
Table 2 Multivariable logistic regression models

\begin{tabular}{lll}
\hline & Odds ratio (95\% CI) & $p$ value \\
\hline Model 1: calculation of scores and positive answers & & \\
Well/Geneva score calculated/not calculated & $0.50(0.18-1.37)$ & 0.18 \\
Positive answer: reason Wells/Geneva score & $3.28(1.24-8.68)$ & 0.017 \\
Positive answer: reason elevated D-dimers & $1.56(0.66-3.70)$ & 0.32 \\
Positive answer: reason fear of missing PE & $0.36(0.14-0.92)$ & 0.033 \\
Positive answer: reason request of the patient/relatives & $2.18(0.62-7.65)$ & 0.22 \\
Positive answer: reason fear of being sued & $0.47(0.11-2.05)$ & 0.32 \\
Model 2: scale parameters & $2.46(1.26-4.77)$ & 0.008 \\
Scale parameters: reason Wells/Geneva score & $3.40(1.19-9.72)$ & 0.023 \\
Scale parameters: reason elevated D-dimers & $0.27(0.10-0.71)$ & 0.008 \\
Scale parameters: reason fear of missing PE & $3.48(0.21-57.64)$ & 0.38 \\
Scale parameters: reason request of the patient/relatives & $0.37(0.03-5.38)$ & 0.47 \\
Scale parameters: reason fear of being sued & \\
\hline
\end{tabular}

The outcome measure was positive CTPA. For scale parameters from 0 to 10 , the odds ratio (OR) was expressed as the ratio of the odds from the 3rd to the 1 st quartile ( 75 and 25 percentile, respectively), representing a typical above average to a typical below average value. A positive answer was defined as any marked number from 1 to 10 on the scale and scale parameters for the reason "Wells/Geneva score" were associated with positive CTPA, but not just the fact of calculation of the Wells/Geneva score. Scale parameters, but not a positive answer for the reason "elevated D-dimers", were associated with positive CTPA (Table 2).

\section{Pretest probability}

A pretest probability for PE before CTPA was defined in 137 out of 140 questionnaires. There were 40 high, 67 intermediate, and 30 low pretest probabilities. A calculated Wells/Geneva score was declared in 97 questionnaires $(71 \%)$ to be a contributing factor in defining pretest probability. High pretest probability for PE was associated with positive CTPAs compared to low pretest probability $(62.5$ vs. $10 \%$, OR $15,95 \% \mathrm{CI}$ $3.87-58.08, p<0.001)$, whereas the proportion of positive CTPAs for intermediate pretest probability was $13 \%$. The proportion of positive CTPAs was much the same for high, intermediate, or low pretest probability if the pretest probability was estimated by calculating the Wells/Geneva score or if the score was not calculated. A positive answer for "fear of missing PE" was declared in $73 \%$ of questionnaires if the pretest probability was low, $55 \%$ if it was intermediate, and $43 \%$ if it was high. In 25/140 (18\%) questionnaires, low or intermediate pretest probability was declared and CTPA was performed, although the D-dimer test was not elevated or not performed.

Positive CTPAs in the study and comparison periods

During the study period, the proportion of positive CTPAs was not significantly higher than in the comparison period, but the proportion of positive CTPA was significantly higher if a questionnaire was completed compared to the comparison period and than if a questionnaire was not completed. There was no significant difference in the proportion of positive CTPAs if no questionnaire was completed, compared to the comparison period. These results were similar for those CTPAs evaluating patients for PE only and not for an additional disease (Table 3).

\section{Discussion}

Our findings indicate that factors reflecting defensive behavior such as "fear of missing PE" were the reason for ordering CTPA in more than half of the orders, at a similar frequency to factors reflecting evidence-based medicine, such as the Wells/Geneva score. Factors like "request from the patient or his/her relatives" or "fear of being sued" played a minor role. This corresponds to a small number of prosecutions of physicians in Switzerland and stands in contrast to the USA where the risk of facing a malpractice claim is high [10]. Scale parameters of "elevated D-dimers" as a reason for a CTPA were associated with positive CTPA, although elevated D-dimers are not specific for PE [11]. However, our D-dimer test, if not elevated, can safely rule out PE in patients with low or intermediate probability for PE [12]. Thus, if used together with pretest probability, the reason "elevated D-dimer" can be seen as an element of evidence-based behavior.

After training in using evidence-based guidelines in diagnosing PE, the proportion of positive CTPAs increased, particularly in the group of patients for whom a questionnaire was completed. There were more patients with risk factors for PE in this group, indicating more careful selection of candidates for CTPA. Thus, our study 
Table 3 Proportions of positive CTPAs during study and comparison periods

\begin{tabular}{|c|c|c|c|}
\hline & Positive CTPA— $n(\%)$ & Odds ratio $(95 \% \mathrm{CI})$ & $p$ value \\
\hline \multicolumn{4}{|l|}{ All CTPAs $(n=900)$} \\
\hline All during study period vs. comparison period & $63 / 328(19.2 \%)$ vs. $83 / 572(14.5 \%)$ & $1.40(0.98-2.0)$ & 0.067 \\
\hline With questionnaire vs. comparison period & $37 / 140(26.4 \%)$ vs. $83 / 572(14.5 \%)$ & $2.12(1.36-3.29)$ & $<0.001$ \\
\hline Without questionnaire vs. comparison period & 26/188 (13.8 \%) vs. 83/572 (14.5\%) & $0.95(0.59-1.52)$ & 0.82 \\
\hline With questionnaire vs. without questionnaire & $37 / 140(26.4 \%)$ vs. $26 / 188(13.8 \%)$ & $2.24(1.28-3.91)$ & 0.0047 \\
\hline \multicolumn{4}{|l|}{ CTPAs, evaluation for PE only $(n=509)^{\mathrm{a}}$} \\
\hline All during study period vs. comparison period & $37 / 173(21.4 \%)$ vs. $55 / 336(16.4 \%)$ & $1.39(0.87-2.21)$ & 0.17 \\
\hline With questionnaire vs. comparison period & $29 / 85(34.1 \%)$ vs. $55 / 336(16.4 \%)$ & $2.65(1.55-4.51)$ & $<0.001$ \\
\hline Without questionnaire vs. comparison period & $8 / 88(9.1 \%)$ vs. $55 / 336(16.4 \%)$ & $0.51(0.23-1.12)$ & 0.092 \\
\hline With questionnaire vs. without questionnaire & $29 / 85(34.1 \%)$ vs. $8 / 88(9.1 \%)$ & $5.18(2.20-12.2)$ & $<0.001$ \\
\hline
\end{tabular}

Odds ratios, $95 \% \mathrm{CI}$, and $p$ values were calculated by logistic regression. A difference with a $p$ value of less than 0.05 was considered to be significant

Positive CTPA evidence of central, paracentral, segmental, or subsegmental embolism of the pulmonary artery in CTPA; without questionnaire no questionnaire completed during study period

might have directed physicians towards more evidencebased behavior by making them familiar with scores as a result of training, by considering pretest probabilities, and by documentation of reasons for ordering CTPA. However, although training was provided, the Wells/Geneva score was a reason for CTPA in half of the orders only, and guidelines were not respected in $18 \%$ of orders (i.e., CTPA was performed in cases of low or intermediate pretest probability and the D-dimer test was either not elevated or not performed). This corresponds to the results of an international survey of emergency physicians analyzing awareness and use of the Canadian CT head rules and Canadian cervical spine rules; this found that only $12-33 \%$ of physicians were using these guidelines, although the awareness of these guidelines was 31-80\% [13]. This is also analogous to the results of two studies in our emergency department that found that physicians adhered to evidence-based institutional guidelines in only half of the orders of CT of the head in patients with minor brain injury, and only a minority of physicians respected institutional guidelines for radiological tests when diagnosing acute appendicitis [14, 15].

Physicians who had "fear of missing PE", reflecting defensive behavior, had a significantly lower yield of positive CTPA, whereas physicians who considered the Wells/Geneva score to be a reason for CTPA, reflecting evidence-based behavior, had a better yield of positive CTPA, and "fear of missing PE" was present particularly in cases with low pretest probability for PE. This corresponds to a study in evaluating patients with possible acute cardiac ischemia, where fear of malpractice was associated with increased unnecessary hospitalization of low risk patients and increased use of diagnostic tests [16]. However, just the fact that the Wells/Geneva score was calculated was not associated with positive CTPA. But, patients with a high pretest probability for PE were more likely to have a positive CTPA, regardless of a CTPAs that evaluated patients for PE only and not for any additional diseases such as consequences of trauma, cancer, aortic dissection, or pulmonary diseases

whether the pretest probability was estimated by using the Wells/Geneva score, or if it was estimated by considering other criteria (e.g., the Pulmonary Embolism Rule Out Criteria (PERC), including the risk factor oral contraceptive [17]), or the clinical experience of the physician. Thus, the Wells/Geneva score should be seen as one possible tool for estimating the pretest probability of PE, among a variety of other factors that influence the estimation of pretest probability, such as all clinical experience and diagnostic skills of the physician.

The role of defensive medicine in driving up healthcare cost is controversial. However, there are estimates that defensive medicine costs the US health-care system up to 45-100 billion dollars annually and is thought to account for 5-25\% of all medical care costs in the USA $[7,9,18,19]$. To reduce health-care cost, the need to reduce the practice of defensive medicine is emphasized, and education of physicians in evidence-based medicine is requested [19].

Our study had several limitations: First, questionnaires were completed in only $43 \%$ of the cases that a CTPA was ordered. This is in line with a meta-analysis of response rates to mail surveys published in medical journals, where the response rate of physicians was $54 \pm 17$ (mean $(\%) \pm$ SD) [20]. Second, a selection bias for completing the questionnaire is conceivable, and the possibility cannot be excluded that physicians were influenced by a high probability of $\mathrm{PE}$ in deciding to complete the questionnaire. However, less than a third of questionnaires indicated high probability. Thus, it does not seem that the high yield of positive CTPAs of physicians who filled out questionnaires is just a result of selection bias. Third, we are not able to prove that the higher proportion of positive CTPAs during the study period was due to training and completing questionnaires. Different physicians were working during the time periods, so the difference could be due to better qualified 
physicians. However, there was no difference in positive CTPAs between the comparison period and the group without a completed questionnaire, indicating that physicians during the comparison period and physicians who did not fill out questionnaires possessed similar diagnostic skills. Finally, this study was performed at a single institution. Thus, our findings may not be generalizable.

In conclusion, reasons for ordering CTPA reflecting defensive behavior such as "fear of missing PE" were frequent in the setting of diagnosing PE in the emergency department, and were associated with a decreased odds of positive CTPA. We believe that physicians' defensive behavior is modifiable by teaching evidence-based guidelines to estimate pretest probabilities and by reminding physicians of their reasons for ordering diagnostic tests, e.g., by documentation of motivations. Future efforts should focus on knowledge translation and the implementation of existing guidelines.

Acknowledgments We thank Schötzau and Simmen, Statistical Consulting, Basel, Switzerland, for statistical analysis, and Rodney Yeates PhD for reviewing the manuscript. We thank Maja Weisser MD, and Luigia Elzi MD, Department of Infectious Diseases and Hospital Epidemiology, University Hospital Basel, Switzerland, for their valuable ideas.

Conflicts of interest None.

\section{References}

1. Oger E (2000) Incidence of venous thromboembolism: a community-based study in Western France. EPI-GETBP Study Group. Groupe d'Etude de la Thrombose de Bretagne Occidentale. Thromb Haemost 83:657-660

2. Spencer FA, Emery C, Lessard D, Anderson F, Emani S, Aragam J, Becker RC, Goldberg RJ (2006) The Worcester venous thromboembolism study: a population-based study of the clinical epidemiology of venous thromboembolism. J Gen Intern Med 21:722-727

3. Goldhaber SZ, Visani L, De Rosa M (1999) Acute pulmonary embolism: clinical outcomes in the International Cooperative Pulmonary Embolism Registry (ICOPER). Lancet 353:1386-1389

4. Agnelli G, Becattini C (2010) Acute pulmonary embolism. N Engl J Med 363:266-274

5. Wells PS, Anderson DR, Rodger M, Ginsberg JS, Kearon C, Gent M, Turpie AG, Bormanis J, Weitz J, Chamberlain M, Bowie D, Barnes D, Hirsh J (2000) Derivation of a simple clinical model to categorize patients probability of pulmonary embolism: increasing the models utility with the SimpliRED D-dimer. Thromb Haemost 83:416-420

6. Klok FA, Mos IC, Nijkeuter M, Righini M, Perrier A, Le Gal G, Huisman MV (2008) Simplification of the revised Geneva score for assessing clinical probability of pulmonary embolism. Arch Intern Med 168:2131-2136

7. Hermer LD, Brody H (2010) Defensive medicine, cost containment, and reform. J Gen Intern Med 25:470-473
8. Studdert DM, Mello MM, Sage WM, DesRoches CM, Peugh J, Zapert K, Brennan TA (2005) Defensive medicine among high-risk specialist physicians in a volatile malpractice environment. JAMA 293:2609-2617

9. Khemani RG, Sward K, Morris A, Dean JM, Newth CJ (2011) Variability in usual care mechanical ventilation for pediatric acute lung injury: the potential benefit of a lung protective computer protocol. Intensive Care Med 37:1840-1848

10. Jena AB, Seabury S, Lakdawalla D, Chandra A (2011) Malpractice risk according to physician specialty. N Engl J Med 365:629-636

11. Di Nisio M, Squizzato A, Rutjes AW, Buller HR, Zwinderman AH, Bossuyt PM (2007) Diagnostic accuracy of D-dimer test for exclusion of venous thromboembolism: a systematic review. J Thromb Haemost 5:296-304

12. Carrier M, Righini M, Djurabi RK, Huisman MV, Perrier A, Wells PS, Rodger M, Wuillemin WA, Le Gal G (2009) VIDAS D-dimer in combination with clinical pre-test probability to rule out pulmonary embolism. A systematic review of management outcome studies. Thromb Haemost 101:886-892

13. Eagles D, Stiell IG, Clement CM, Brehaut J, Taljaard M, Kelly AM, Mason S, Kellermann A, Perry JJ (2008) International survey of emergency physicians' awareness and use of the Canadian cervical-spine rule and the Canadian computed tomography head rule. Acad Emerg Med 15:1256-1261
14. Rohacek M, Albrecht M, Kleim B, Zimmermann H, Exadaktylos A (2012) Reasons for ordering computed tomography scans of the head in patients with minor brain injury. Injury. doi:10.1016/j.injury.2012.01.001

15. Boehnert MU, Zimmermann H, Exadaktylos AK (2009) O knowledge, where art thou? Evidence and suspected appendicitis. J Eval Clin Pract 15:1177-1179

16. Katz DA, Williams GC, Brown RL, Aufderheide TP, Bogner M, Rahko PS, Selker HP (2005) Emergency physicians' fear of malpractice in evaluating patients with possible acute cardiac ischemia. Ann Emerg Med 46:525-533

17. Singh B, Parsaik AK, Agarwal D, Surana A, Mascarenhas SS, Chandra S (2011) Diagnostic accuracy of pulmonary embolism rule-out criteria: a systematic review and meta-analysis. Ann Emerg Med. doi: 10.1016/j.annemergmed.2011.10.022

18. Mello MM, Chandra A, Gawande AA, Studdert DM (2010) National costs of the medical liability system. Health Aff (Millwood) 29:1569-1577

19. Malach M, Baumol WJ (2012) Opportunities for cost reduction of medical care: part 3. J Community Health. doi:10.1007/s10900011-9534-8

20. Asch DA, Jedrziewski MK, Christakis NA (1997) Response rates to mail surveys published in medical journals. J Clin Epidemiol 50:1129-1136 